FERMILAB -Conf-91/202-A

July, 1991

\title{
CONVERTING NEUTRON STARS INTO STRANGE STARS
}

\author{
A. V. Olinto \\ Department of Astronomy and Astrophysics \\ Enrico Fermi Institute, \\ University of Chicago, Chicago, IL 60637 \\ NASA/Fermilab Astrophysics Center \\ Fermi National Accelerator Laboratory \\ P.O. Box 500, Batavia, IL 60510-0500
}

\begin{abstract}
If strange matter is formed in the interior of a neutron star, it will convert the entire neutron star into a strange star. We review the proposed mechanisms for strange matter seeding and the possible strange matter contamination of neutron star progenitors. We discuss the conversion process that follows seeding and the recent calculations of the conversion timescale.
\end{abstract}

to appear in the Proccedings of "Strange Quark Matter in Physics and Astrophysics," Aarhus, Denmark 1991.

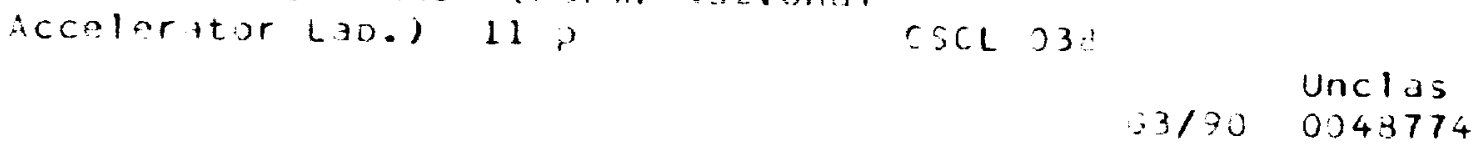



When a neutron and a stable strangelet meet the neutron is readily absorbed, while a proton can coexist with a strangelet due to the Coulomb barrier between them. ${ }^{1,2}$ Therefore, if a stable strangelet (a strangelet with baryon number greater than or equal to some minimum baryon number for strange matter stability, $A_{\min }$ ) makes its way to the neutron rich regions of a neutron star, it will grow by absorbing neutrons and, eventually, convert most of the neutron star into a strange star. ${ }^{1,3,4,5,6}$ The conversion of a neutron star into a strange star is quite a spectacular event: one stable strangelet can seed the conversion process that would liberate about $10^{52}$ ergs in binding energy (for a strange matter bound by $10 \mathrm{MeV}$ ).

The only region of a neutron star that could survive this conversion is the outer crust, since densities there are below the density where neutrons drip out of nuclei. The Coulomb barrier between strange matter and the ions in the outer crust forms a gap of a few hundred fermis, which is sufficient to support the entire outer crust of the neutron star above the strange matter interior. ${ }^{4}$

In what follows, I first review the possible seeding mechanisms of strange matter in neutron stars and discuss the implications of an abundance of strangelets in the interstellar medium. Then, I will discuss the propagation of the conversion front and the possible observables of such a remarkable event.

\section{SEEDING MECHANISMS}

A variety of different seeding mechanisms have been suggested so far. ${ }^{3,6}$ They can be divided into two main categories: primary mechanisms, in which the seed is formed inside the neutron star; ${ }^{7}$ and secondary mechanisms, in which the seed comes from the interstellar medium. ${ }^{7,8}$ Obviously, there can only be secondary processes, if there were some primary ones before.

\subsection{Primary Processes}

The formation of a strangelet is much more likely in a neutron star than in ordinary nuclei. The central pressures in a neutron star may be sufficient for a phase transition from neutron matter to two-flavor quark matter to occur. Two-flavor quark matter can then easily decay into the lower energy strange matter via weak interactions. ${ }^{1,3,4}$ (Unlike the case for nuclei, here the formation of many strange quarks does not need to be simultaneous.) The possible existence of quark matter in the inner core of neutron stars has been widely studied, but no firm conclusion can be reached at present. ${ }^{\top}$

Another possible way of forming a seed of strange matter is via an agglomeration of $\Lambda$ particles. At very high densities $\left(\rho \geq 1.6 \times 10^{15} \mathrm{gm} / \mathrm{cm}^{3}\right)$, the neutron chemical potential becomes greater than the $\Lambda$ mass and $\Lambda$ 's start to appear. ${ }^{\circ}$ Strangelets can then form 
directly by the overlap of $A_{\min } \Lambda$ 's. The probability of $A_{\min } \Lambda$ 's overlapping is roughly $P\left(A_{\min }\right) \simeq\left(n_{\Lambda} V_{A_{\min }}\right)^{A_{\min }}$, where $n_{\Lambda}$ is the number density of $\Lambda$ 's and $V_{A_{\min }}$ is the volume of a strangelet with $A=A_{\min }$. The exact value of $A_{\min }$ (and, therefore, of $V_{A_{\text {min }}}$ ) is very uncertain, but it is expected to be of the order of 100 or larger. Then, $P\left(A_{\min }\right)$ jumps from negligible $\left(\sim 10^{-100}\right)$ to $\sim 1$, when $n_{A} V_{A_{\min }}$ varies from 0.1 to 1 . The outcome of this process is hard to determine due to the the strong dependence on two uncertain parameters: the density of $\Lambda$ 's reached inside neutron stars and the value of $A_{\min }$.

Hadronic condensates with strangeness can also be present in neutron stars at high enough densities. Kaon condensates ${ }^{10}$ and dibaryon condensates ${ }^{11}$ may occur in neutron stars for certain ranges of parameters. Both types of condensate would facilitate the formation of strange matter seeds.

Strange matter seeds may also be formed through the burning of neutrons into strangelets. ${ }^{4}$ Neutron stars are born hot, so neutrons can be heated into higher energy states such as low baryon number strangelets, which can then form higher baryon number (lower energy) strangelets. The process is analogous to chemical burning through an activation barrier. In ref. 4, we estimated the rate at which strangelets are made per unit volume. Again, we find that the rate varies from "extremely large" to "vanishingly small," with a small variation of the temperature and of the mass of the low baryon number strangelets (for example, a factor of two in the parameters changes the rate from $\sim 10^{45} / \mathrm{cm}^{3} \mathrm{~s}$ to $\left.\sim 10^{-125} / \mathrm{cm}^{3} \mathrm{~s}\right)$.

Finally, a more contrived way to form strangelets is via neutrino sparking. ${ }^{4}$ Here, an ultra-high energy neutrino $\left(E_{\nu} \sim 10^{14} \mathrm{MeV}\right)$, the secondary from an ultra-high energy cosmic ray, penetrates a neutron star and scatters inelastically off a quark, depositing most of its energy in a small volume. This forms a hot quark-gluon plasma, with $s \bar{s}$ pairs. If strangeness separation is efficient and cooling is fast enough, strangelets could be formed. This process shares some of the difficulties of forming stable strangelets (low entropy states) in heavy ion collisions (high entropy environments). On the other hand, it could possibly be tested by future quark-gluon plasma experiments.

\subsection{Secondary Processes}

If any of the above primary seeding processes (or some other we have not envisioned yet) can convert some neutron stars into strange stars, it is likely that all young neutron stars are strange stars. This statement is based on recent estimates, by Friedman and Caldwell, of the abundance of strangelets in the interstellar medium due to the coalescence of binary systems of two strange stars or of a strange star and a black hole.

If strange stars exist, they will naturally be found in binary systems. Some fraction, $f$, of strange stars will be in binaries with other strange stars or with black holes. These 
systems will eventually coalesce violently and in the process, some fraction of the lower density strange star will be ejected into the interstellar medium. (Neutron star-strange star binary systems will most likely end in the disruption of the neutron star not the strange star, since strange stars are expected to be more dense than neutron stars. $)^{8}$ Before the final coalescence, the more dense companion will tidally strip some mass from the lower density strange star. Typical sizes for strange matter lumps that are stripped can be estimated by balancing the tidal pull of the companion star against the surface tension of the strange star. For a $1 M_{\odot}$ companion at $30 \mathrm{~km}$ separation, the typical stripped strange matter lump is around $10^{45}$ in baryon number. ${ }^{3}$ Lumps this large will not contaminate the interstellar medium effectively. If they escape the binary system, they will most likely escape the galaxy all together. If they stay bound to the binary system, these lumps will orbit the system and will collide with each other from time to time. The collision of these strange matter satellites will break them down into smaller lumps. Of these smaller lumps, those with $A \leq 10^{6}$ remain in their parent galaxy confined by the galactic magnetic field, and will become part of the interstellar medium. ${ }^{8}$

Neutron star coalescence studies give estimates of around $0.1-0.01 M_{\odot}$ of ejected material per coalescence. The coalescence rate for neutron stars today in our galaxy is estimated to be around $10^{-4} / \mathrm{yr}$. We can assume that roughly the same rate should apply to strange stars. ${ }^{8}$ If we further assume that this rate has not changed significantly for a billion years, than a lower limit on the number density of strangelets in our galaxy after a billion years is:

$$
n_{s}\left(A \leq 10^{6}\right) \geq f \frac{10^{-4}}{y r} 10^{9} y r f_{6} \frac{0.01 M_{\odot}}{10^{6} m_{p}} \frac{1}{10^{13} L Y^{3}} \simeq f f_{6} 10^{41} / L Y^{3},
$$

where $f_{6}$ is the fraction of the $0.01 M_{\odot}$ ejected in strangelets with $A \leq 10^{\circ}$ per coalescence and the factor $f$ relates to the fraction of strange star-strange star and strange star-black hole binaries.

The strangelets in the interstellar medium can, in principle, seed previously formed neutron stars, ${ }^{4}$ but the probability for this event will depend on a fine tuning of $f$ and $f_{6}$, given that the volume of a neutron star is around $10^{-36} L Y^{3}$. These strangelets will be much more effective in seeding young neutron stars by contaminating their progenitor stars which are formed from a region with a volume $\simeq L Y^{3}$ in the interstellar medium. (Friedman and Caldwell ${ }^{B}$ expect $f$ and $f_{B}$ not far from unity.) If there have been strange stars in binary systems for the last billion years, the progenitors of recent supernovae (formed around ten million years ago) will have been contaminated from birth by strangelets in the interstellar medium. Young neutron stars (like the Crab and Vela) would have been formed with strangelet seeds already in them and would have become strange stars. 
Friedman and Caldwell used this argument together with the notion that strange stars cannot exhibit glitch phenomena (present in young pulsars like Crab and Vela) to rule out the existence of strange matter. This strong conclusion could bring this conference to an early end, were it not for some loopholes.

First, strange matter can be the ground state of hadronic matter, but may never be formed in neutron stars (if none of the primary processes occur). In this case, strange matter has no astrophysical implications but could possibly be studied the laboratory. Second, the notion that strange stars cannot glitch is based on a very simplified and approximate model for stars composed of strange matter and should not be taken as final. Third, the event responsible for the creation of smaller lumps, i.e., the collision of large baryon number satellites, might lead to $f_{\theta} \ll 1$. The smaller strange matter nuggets might all evaporate into neutrons, for example.

Two aspects of the two-component starquake model for pulsar glitches challenge the strange star scenario. ${ }^{12}$ (There have been models proposed where the glitch event originates in the magnetosphere, in which case strange stars are on equal footing with neutron stars.) The first is the need for a crust 100 times more massive (i.e., the outer plus the inner crusts of neutron stars) than the thin outer crust of strange stars, in order to account for the observed spin-down rate. The second ingredient of the phenomenological two-component glitch model that challenges a strange star pulsar model is the need for a neutral superfluid to explain the post-glitch relaxation behaviour.

In principle, the answers to the questions of the stability of strange matter and the structure of high density matter (as well as the understanding of nucleons) lie probably in QCD. Unfortunately, we are not able to extract this information at present, and need to rely on simplified models that describe different aspects of the strong interactions. Our picture of strange stars is based on the bag model, a simplified approach to the complexity of a strange quark matter system. The lack of internal structure in strange stars may be physically correct or may be due to our oversimplification.

Strange stars may have a more complex internal structure than the simple outer crustcore separation. Within quark matter, different correlations may arise at different densities. For example, in a model based on constituent quarks, Donoghue and Siteesh ${ }^{13}$ showed that diquark states may be favored at densities just above the quark-hadron phase transition; in a bag model with massless quarks, diquark states may condense at higher densities, in analogy to dibaryon states in neutron stars. ${ }^{11}$ (Benvenuto et al. ${ }^{14}$ suggested a strange pulsar model making use of another hypothetical state, that of quark-alphas.)

The need for a neutral superfluid also challenges the possibility of a strange matter interior for pulsars. A neutral superfluid in quark matter seems less likely than some 
additional structure. One possibility is that of a neutral pion-like condensate of quarkantiquark pairs, but this has not been seriously explored yet.

Pulsar glitch constraints on strange stars are a very serious challenge to the strange matter hypothesis. Given the present state of affairs, however, a firm conclusion on this issue at awaits observational confirmations of pulsar glitch models and tests of neutron star equations of state, and theoretical or experimental insights into the existence and possible composition of strange quark matter. The lack of a strange star pulsar glitch model may be due to physics or due to lack of imagination.

Finally, the collision of two strange matter satellites orbiting a coalescing binary system might have a different outcome than a production peak of strangelets with $A \simeq A_{\min }$. $A_{\min }$ strangelets may be fragile in high temperature and high entropy environments. ${ }^{16,16,17}$

\section{THE CONVERSION PROCESS}

If we assume that seeding of strange matter has occurred inside a neutron star we can study the consequent conversion of the rest of the star. The first attempt to study the conversion timescale (ref. 3) was conceptually reasonable, but the quantitative results were off by several orders of magnitude. A more complete treatment of the conversion process was given in ref. 6 , with subsequent studies done in refs. 18,19 , and 20 . I'll review this approach and the more recent studies and conclude by discussing the observable consequences of this conversion process.

In general, for simplicity, the strong coupling constant and the strange quark mass are neglected, and with them the small fraction of electrons present. In this case, charge neutrality gives the following relation between the baryon number density, $n$, and the number density of each quark $n_{q}(q=u, d, s): n_{u}=n$ and $n_{d}+n_{\varepsilon}=2 n$. In equilibrium, $\mu_{d}=\mu_{\text {e }}$ and the three number densities are equal, $n_{u}=n_{d}=n_{0}$. Out of equilibrium, there is only one free parameter. (In this picture, $\mu_{u}$ is constant, so we can set $\mu_{u} \equiv \mu \simeq$ $300 \mathrm{MeV}$.) Another simplifying assumption used is that neutron star matter is composed only of neutrons.

The conversion process of neutron matter to strange matter is simpler when viewed from the rest frame of the conversion front. The volume over which strange matter equilibrates was shown ${ }^{18}$ to be much smaller than that of the total strange-matter region, so that the problem can be treated one-dimensionally. In this frame the front can be set at $x=0$, neutron matter is at $x<0$, strange matter at $x>0$, and, asymptotically $(x \rightarrow \infty)$, strange matter is in equilibrium. The region of small positive $x$ has an excess of down quarks relative to strange quarks due to the flux of neutrons $(u d d)$ at $x=0$. The excess down quarks will convert into strange quarks via the weak process $d+u \rightarrow s+u$, as long 
as $\mu_{d}>\mu_{\Omega_{1}}\left(n_{d}>n_{o}\right)$. For large positive values of $x$ the system is close to equilibrium, so $\mu_{d} \simeq \mu_{\text {, and }} n_{d} \simeq n_{a} \simeq n_{u}$.

In terms of a variable $a$ defined by $a(x)=\frac{n_{d}(x)-n_{d}(x)}{n_{d}+n_{d}}$, we can write: $a(x<0)=1$, and, as $x \rightarrow \infty, a(x) \rightarrow 0$. As $x \rightarrow 0$, from the strange matter side $(x>0), a \rightarrow a_{0}$, where $a_{0}$ is the maximum value of $a$ for which strange matter is stable. The value of $a_{0}$ corresponds to the minimum number density of strange quarks such that strange matter is stable. In our scenario, the conversion of neutron matter to strange matter is direct and cannot proceed unless the strange matter next to the conversion front is stable. [It is possible that an activation-type process happens instead, in which two-flavor quark matter is maintained between the neutron matter and the strange matter regions by the energy released in the conversion process. This possibility has not been fully explored at the present.] The limit $a_{0} \rightarrow 0$ corresponds to the case where only strange matter with $n_{d}=n$, can be stable. Then the front cannot move, given that the swallowed neutrons do not have $n_{d}=n_{.}$. The case where $a_{0} \rightarrow 1$ corresponds to stability of two-flavor quark matter; the neutrons can all convert instantaneously. Two-flavor quark matter is known to be unstable; nuclei with baryon number $A$ are made of $A$ nucleons and not of $3 A$ quarks in a single bag. Therefore we expect $0<a_{0}<1$.

The conversion process can be thought of as a fluid of excess down quarks coming from $x<0$ at a velocity equal to that of the front and asymptotically (at $x \gg 0$ ) becoming equilibrium strange matter $(a=0)$. The transformation occurs via two main processes: the conversion of down quarks into strange quarks via $d+u \rightarrow s+u$ (in the region where $\left.\mu_{d}>\mu_{a}\right)$ and the diffusion of the strange quarks from $x \gg 0$ to $x \rightarrow 0$.

These two processes combined give a differential equation for $a(x)$ :

$$
D a^{\prime \prime}-v a^{\prime}-R(a)=0
$$

where $a^{\prime} \equiv \frac{d a}{d x}, D$ is the diffusion coefficient for the strange quarks, and $R(a, T)$ is the rate at which the weak process $u+d \rightarrow u+s$ converts down quarks into strange quarks. The boundary conditions for $a(x)$ are: $a(0)=a_{0}, a(x \rightarrow \infty) \rightarrow 0$, and conservation of baryon number through the conversion front gives: $a^{\prime}(0)=-v\left(1-a_{0}\right) / D .^{\circ}$

The velocity $v$ at which the front moves, is determined by solving the differential equation (2) and imposing the above (overdetermined) boundary conditions; only a special value for $v$ lets all the boundary conditions be satisfied.

The rate, $R$, can be calculated by integrating the square of the matrix element for the process $d+u \rightarrow s+u$ over the phase space of the four particles with the appropriate fermi factors. In the zero-temperature, small $a$ limit, we get: ${ }^{0,19}$

$$
R(a) \simeq \frac{16}{15 \pi^{3}} G_{F}^{2} \cos ^{2} \theta_{c} \sin ^{2} \theta_{c} \mu^{5}\left(\frac{a}{3}\right)^{3}
$$


This can be written as: $R(a) \simeq \frac{a^{3}}{\tau}$, where $\tau \simeq 3.4 \times 10^{-8} \mathrm{sec}$.

Substituting the above $R(a)$ into (2) and imposing the boundary conditions we can find $v\left(a_{0}, r, D\right)$ numerically. The solution can be well approximated by the following analytic expression: ${ }^{\circ}$

$$
v \simeq \sqrt{\frac{D}{\tau} \frac{a_{0}^{4}}{2\left(1-a_{0}\right)}} .
$$

The precise value of $a_{0}$ cannot be well determined due to the uncertainties present in strong-interaction calculations. Reasonable values for $a_{0}$ lie in the range $0<a_{0} \leq 0.5{ }^{2}$ where larger values of $a_{0}$ correspond to stability of strange matter with fewer strange quarks.

The diffusion coefficient is roughly equal to a third of the average speed of the quarks times their mean free path, $\lambda$, in equilibrium strange matter. The quarks move at nearly the speed of light, and their mean free path can be estimated by finding the rate at which they scatter off each other. The temperature dependence of the scattering rate can be determined by the phase-space integral of the fermi factors and the energy-momentum conservation delta function which give a factor of $\left(\frac{T}{\mu}\right)^{2}$ (for $T \ll \mu$ ). A reasonable estimate for the mean free path is $\lambda \simeq 1 \mathrm{fm}\left(\frac{\mu}{T}\right)^{2}$. Therefore,

$$
D=\frac{\bar{v} \lambda}{3} \simeq 10^{-3} \frac{\mathrm{cm}^{2}}{s}\left(\frac{\mu}{T}\right)^{2}
$$

and

$$
v \simeq 2 m / s\left(\frac{\mu}{T}\right) \sqrt{\frac{a_{0}^{4}}{2\left(1-a_{0}\right)}} .
$$

For $a_{0}=0.5, \mu=300 \mathrm{MeV}$, and $T=10 \mathrm{MeV}$, the conversion speed is $v \simeq 15 \mathrm{~m} / \mathrm{s}$, which converts a $10 \mathrm{~km}$ neutron star in about one minute.

The conversion process is very sensitive to the degree of strange matter stability (through $a_{0}$ in (6)), and the temperature at which the conversion is taking place. Note that a minute after its birth a neutron star has cooled well below $10 \mathrm{MeV}, 9$ but the conversion process can release enough energy to reheat the material around the front to equivalent temperatures. The effect of the energy released by the conversion as well as the finite temperature corrections to $R(a, T)$, have been neglected in the model described above. Recently, Heiselberg et al. ${ }^{19}$ have shown that for barely flammable strange matter (i.e., small $a_{0}$ ) the finite temperature corrections to $R(a, T)$ (which are proportional to $\left.a T^{2}\right)$ are at least as important as the $a^{3}$ term. They estimate a conversion timescale of about a minute for small $a_{0}$ (with $\mu=300 \mathrm{MeV}$ and $T=10 \mathrm{MeV}$ ).

Another recent development in the study of the conversion process is the work by Olensen and Madsen. ${ }^{20}$ They have studied the dependence of the conversion timescale on 
temperature, bag constant, and mass of the initial neutron star. They also find a wide range of timescales. If we fix the temperature by the energy released in the conversion, the range can be narrowed; for example, if $T=10 \mathrm{MeV}$, the timescales vary roughly between one and ten minutes.

More progress in understanding this conversion process is "in the works". Baym et al. ${ }^{21}$ studied numerically the general non-linear problem for large $a_{0}$ and $T$. Heiselberg, Madsen, and Olensen ${ }^{22}$ are also studying this process more carefully and seem to have found an extra factor of 24 in the expression for $R(a, T)$. So stay tuned.

Before discussing the possible observable consequences of such a conversion, let me discuss another issue that has attracted some attention on this subject. Horvath et al. ${ }^{18}$ have studied the stability of the conversion front and concluded that the front will be hydrodynamically unstable. They speculate that this instability will lead to the formation of a detonation front. The mechanism by which a detonation front could be sustained is not very clear, given that there are limitations to how fast strangeness can be formed and supplied to the detonation front due to the slowness of the weak interactions. These instabilities might instead slow down the conversion process. ${ }^{23}$ This issue is not yet settled. Benvenuto et al. ${ }^{24}$ suggest that if a detonation front is formed, the energy released by the conversion of neutron stars into strange stars could come to the aid of the failed supernova explosion simulations. However, it is not clear how the energy released in the conversion could get transferred to the supernova shock front way into the envelope of the progenitor (far from the neutron star), as pointed out by P. Haensel during this meeting.

In any case, a more careful study of the conversion process which addresses the reheating due to the strange matter binding energy, the possibility of activating the conversion via two-flavor quark matter regions, and the consequences of the hydrodynamical instabilities should be done.

The conversion of a neutron star into a strange star is more likely to happen just after a neutron star is born. The conditions for primary seeding of strange matter are better at this stage and the progenitor might have some strangelet contamination already. In this case, an observable consequence would be an extra neutrino flux produced thermally at the conversion front. ${ }^{6}$ With the possibility of detecting the neutrinos from a supernova explosion in our galaxy, we will be able to confirm or rule out such a conversion. In general, the conversion would be seen as a longer tail on the neutrino pulse observed on earth. The exact form of the neutrino emission will depend on more careful studies of the conversion already underway.

If an older neutron star gets converted, the conversion might be observed by a faster (lower $T$ ) neutrino burst accompanied by a gamma ray burst. ${ }^{6}$ 
I would like to thank the organizers and the participants of this workshop on "Strange Quark Matter in Physics and Astrophysics." This work was supported by DOE (DEFG02-90ER40606) and NSF (AST-22595) at the University of Chicago, and by the NASA (NAGW-1340) at Fermilab.

\section{REFERENCES}

1. E. Witten, Phys. Rev. D 30 (1984) 272.

2. E. Farhi and R. L. Jaffe, Phys. Rev. D 30 (1984) 3279.

3. G. Baym, E. Kolb, L. McLerran, T. P. Walker, and R. L. Jaffe, Phys. Letters 160B (1985) 181.

4. C. Alcock, E. Farhi and A. Olinto, Ap. J. 310 (1986) 261.

5. P. Haensel, J.L. Zdunik, and R. Schaeffer, Astron. Astrophys. 160 (1986) 121.

6. A.V. Olinto, Phys. Lett. 192B (1987) 71.

7. C. Alcock and A.V. Olinto, Ann. Rev. Nucl. Part. Sci. 38 (1988) 161, and references therein.

8. J. Friedman and R. Caldwell, to be published in Phys. Lett. B (1991).

9. S. L. Shapiro and S. A. Teulkolsky, Black Holes, White Dwarfs, and Neutron Stars - The Physics of Compact Objects (Wiley-Interscience Pub., 1983).

10. A.E. Nelson and D.B. Kaplan, Phys. Lett. 192B (1987) 193.

11. A.V. Olinto, P. Haensel, and J. Frieman, submitted to Phys. Rev. Let. (1991).

12. M.A. Alpar, Phys. Rev. Lett. 58 (1987)2152.

13. J.F. Donoghue and K.S. Sateesh, Phys. Rev. D 38 (1988).

14. O.G. Benvenuto and J.E. Horvath, Phys. Rev. Let. 64 (1990) 713.

15. C. Alcock and E. Farhi, Phys. Rev. D 32 (1985) 1273.

16. C. Alcock, E. Farhi, and A.V. Olinto, Phys. Rev. Lett. 57 (1986) 2088.

17. C. Alcock and A.V. Olinto, Phys. Rev. D 39 (1989) 1233.

18. J.E. Horvath and O.G. Benvenuto, Phys. Lett. 213B (1988) 516. 
19. H. Heiselberg, G. Baym, and C.J. Pethick, in this volume.

20. M.L. Olesen and J. Madsen, in this volume.

21. G. Baym, H. Heiselberg, and C.J. Pethick, to be published.

22. H. Heiselberg, J. Madsen, and M.L. Olensen, in preparation.

23. J. Miller, private communication.

24. O.G. Benvenuto, J.E. Horvath, and H. Vucetich, Int. J. Mod. Phys. A4 (1989) 257. 